\title{
Coordination of metabolism, arousal, and reward by orexin/hypocretin neurons
}

\author{
Natalie J. Michael' and Joel K. Elmquist ${ }^{2}$ \\ IInstitut Universitaire de Cardiologie et de Pneumologie de Québec, Quebec City, Quebec, Canada. Center for Hypothalamic Research, Department of Internal Medicine and Department of Pharmacology, \\ UT Southwestern Medical Center, Dallas, Texas, USA.
}

Orexin/hypocretin neurons located in the lateral hypothalamus play a critical role in the maintenance of arousal and contribute to the regulation of multiple homeostatic and behavioral processes. In this issue of the JCI, Tan and Hang et al. report that feeding a high-fat diet to mice compromised the function of the orexin system, leading to impairments in reward-seeking and active coping mechanisms. The researchers observed changes at the cellular and circuit levels suggesting that reduced excitability of orexin neurons affects behavior through induction of a hypoarousal state.

\section{CNS circuits are altered in obesity}

Obesity represents a growing global public health crisis. In addition to increasing the risk of mortality and the likelihood of suffering other serious diseases, obesity is associated with alterations in several CNS functions. Initial research efforts focused on the consequences of high-fat feeding (HFF) on the neural circuits regulating feeding, metabolism, and cardiovascular functions (1). More recently, interest has shifted to the impact of obesity on cognitive and behavioral processes. Obesity negatively disrupts reward behavior, mood, and arousal $(2,3)$. However, the mechanisms by which obesity perturbs the CNS circuits regulating complex behaviors remain unclear.

An example of a neuronal population that is ideally positioned to link metabolism, arousal, and reward processing is the orexin population, located in the lateral hypothalamus (4-6). Initial reports describing the discreet neuroanatomical location of orexin neurons and their diffuse projections suggested they may regulate multiple physiological processes. Indeed, orexin neurons were quickly recognized for their importance in metabolic regulation and arousal (5, 7-9).
Central administration of orexin was shown to stimulate feeding, and fasting results in upregulation of the orexin precursor peptide (prepro-orexin) (5). In addition, functional loss of the orexin (Hcrt) gene or receptors in mice and dogs results in severe deficits in wakefulness, mirroring the sleep disorder known as narcolepsy $(7,8)$. Moreover, postmortem studies in human subjects indicate that narcolepsy associates with loss of orexin neurons (10).

Subsequent studies linking the orexin system with both feeding and wakefulness suggest that orexin neurons are important for driving increases in arousal and locomotor activity required for food-seeking behaviors (11). This concept further extends to include other motivationally driven behaviors. With connections to the emotion and reward centers in the brain, orexin neurons have since been implicated in reward processing and addictive behaviors $(12,13)$. Therefore, orexin neurons' extensive innervation of the CNS, role in arousal, and ability to act as sensors of the metabolic state make these neurons prime candidates for linking overnutrition and obesity with altered behavior.

\section{Related Article: p. 4985}

Conflict of interest: The authors have declared that no conflict of interest exists.

\section{Effects of obesity on the orexin system}

In this issue of the JCI, Tan and Hang et al. (14) provide exciting results related to the impact of diet-induced obesity (DIO) on orexin neuronal functions and the behavioral responses they regulate. The researchers report that mice fed a high-fat diet developed impairment of glutamatergic neurotransmission to orexin neurons, including decreased probability of neuropeptide release, decreased synaptic potentiation, and decreased dopamine-mediated excitation (Figure 1). In addition to the altered excitatory inputs to orexin neurons, intra- and extrahypothalamic circuit functions were altered. Electrophysiological recordings showed increased spontaneous $\gamma$ oscillations in the lateral hypothalamus and decreased evoked hippocampal $\theta$ power; both neuroanatomical regions represent direct targets of the orexin neurons. Furthermore, these changes were accompanied by decreased reward seeking, demonstrated by reduced cocaineinduced conditioned place preference (CPP) and decreased coping responses to a stressor (forced swim test).

Given the previously recognized role of the orexin neurons in regulating motivationally driven behaviors via increases in arousal $(11,12)$, these results suggest that the diet-induced decrease in excitatory tone to the orexin neurons may reduce arousal and underlie the observed behavioral modifications. To test this theory, Tan and Hang et al. (14) exploited pharmacological and chemogenetic techniques to increase orexin neuron activity and arousal. The researchers selectively activated orexin neurons with excitatory designer receptors exclusively activated by designer drugs (DREADDs). Notably, the DREADDs reversed the downstream neural circuit changes and corrected the decrease in reward seeking and compromised stress coping that occurred with HFF. When the authors treated the mice 

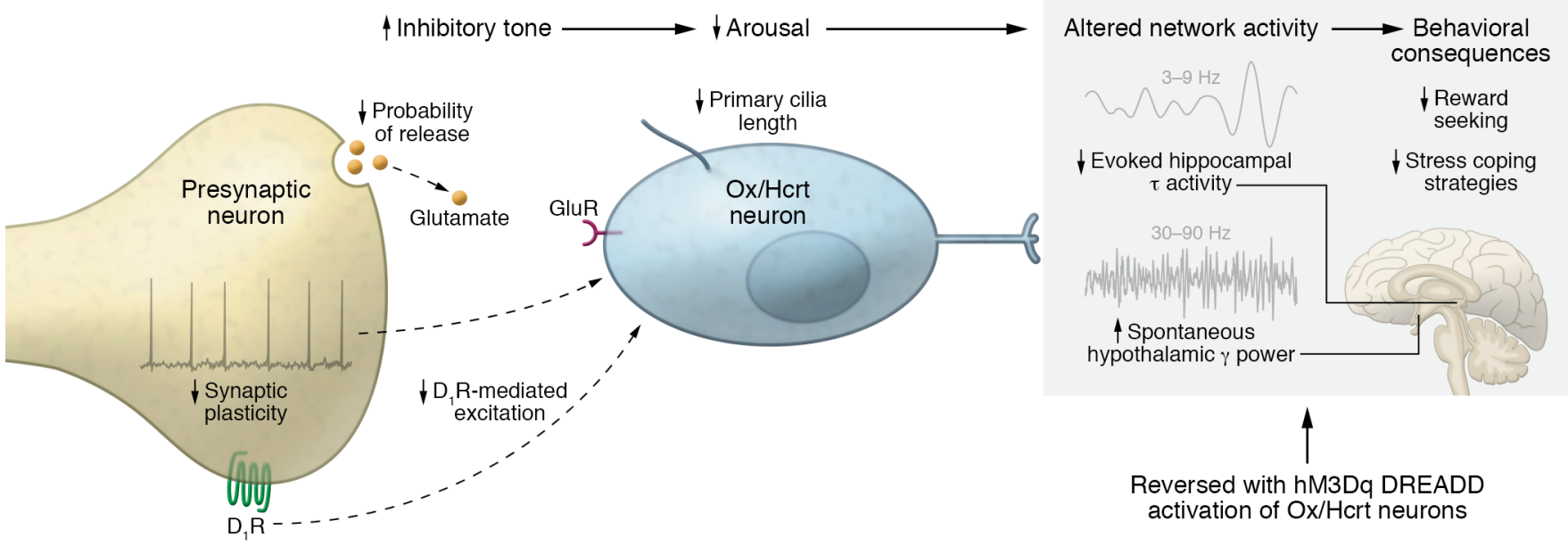

Figure 1. Obesity-induced cellular and circuit level changes in the orexin system are associated with maladaptive behavioral responses. Chronic HFF alters glutamatergic inputs to orexin/hypocretin neurons (Ox/Hcrt), thereby increasing overall inhibitory tone and inducing a state of hypoarousal. Altered cellular activity of orexin neurons influences the activity of downstream neural networks in the lateral hypothalamus and hippocampus and decreases reward-seeking and coping behaviors. Chemogenetic activation of orexin neurons reverses changes in network activity and corrects the behavioral consequences of HFF (14). GluR, glutamate receptor; $D_{1} R$, dopamine 1 receptor; hM3Dq, chemogenic receptor.

with a therapeutic for narcolepsy (modafinil) to enhance alertness, the mice also overcame the obesity-induced stress deficit. Collectively, these findings suggest that hypoarousal mediated by the orexin system may cause the neural circuit and behavioral changes observed with HFF.

\section{Physiological significance and future studies}

Glutamatergic synaptic activity contributes to the basal electrical excitability of orexin neurons (15), and increasing evidence suggests that metabolic status is central in setting this tone. While Tan and Hang et al. (14) demonstrate that chronic HFF decreased signaling at glutamatergic synapses onto orexin neurons, fasting has been shown to have the opposite effect and rapidly promotes excitatory neurotransmission at orexin synapses (16). These synaptic changes are likely crucial for orexin neurons' ability to formulate the appropriate autonomic, endocrine, or behavioral responses according to metabolic status; they also raise questions about how the orexin system may change over time with exposure to HFF. Conveniently, data recently published demonstrates that HFF produces a time-dependent change in orexin neuron synaptic plasticity (17). Short-term HFF (1 day to 1 week) is associated with increased excitatory drive to orexin neurons, which then reverses with longer-term consumption (17). Together, these studies demonstrate that multiple pre- and postsynaptic mechanisms serve to dynamically regulate glutamatergic inputs to orexin neurons, which parallel short- and long-term changes in the metabolic state.

The orexin system adaptation to HFF likely serves to regulate arousal and the motivation for reward relative to metabolic status. However, the paper by Tan and Hang et al. raises additional questions about what is driving the effect. DIO associates with multiple physiological changes, and orexin neurons are responsive to various signals reflective of energy status, including circulating nutrients, endocannabinoids, and hormones $(12,18)$. A large component of the diet-driven effect appears to involve presynaptic mechanisms. Given that leptin administration can prevent fasting-induced synaptic changes at orexin synapses (16), persistently elevated leptin levels may also contribute to the synaptic changes seen with HFF.

Notably, the central melanocortin system innervates orexin neurons, including excitatory inputs from proopiomelanocortin neurons (19). The melanocortin system is crucial in detecting energy status and signals to other arousal centers via perifornical lateral hypothalamic neurons (20). Furthermore, this relationship is bidirectional (21), and connections between orexin neurons and the energy-sensing arcuate nucleus neurons likely contribute to such circuit function changes. These data raise the possibility that multiple signals reflective of metabolic status may influence orexin system function. While it is interesting to speculate, further work is required to determine the precise factors driving the synaptic changes with HFF.

Additional remaining questions relate to the outputs driving the behavioral consequences. Although it is likely that orexin itself mediates the effects, orexin neurons coexpress glutamate, and chemogenic activation of orexin neurons provides limited control over selective release of peptides or fast neurotransmitters (22). Furthermore, both orexin receptor (OxR) subtypes are expressed in regions of the brain implicated in arousal, motivation, and reward (12). While the OxR1 may be more relevant for reward and OxR2 for arousal (13), the contribution of each receptor to the observed effects is unknown. Moreover, the downstream target neurons mediating these effects remain undetermined. Although orexin neurons directly project to the hippocampus and reward systems, they also heavily innervate monoaminergic arousal-promoting neurons (12). This includes the histaminergic neurons of the tuberomammillary nucleus, which are important 
for mediating orexin effects on arousal (23). Histaminergic neurons are also implicated in motivation and goal-directed behaviors and project to many important brain regions regulating these processes, including the ventral tegmental area and the nucleus accumbens (24). These details highlight the potential for orexin neurons to influence complex behaviors in both direct and indirect manners.

The orexin system sits at the intersection of metabolism, reward, and arousal control. The study by Tan and Hang et al. (14) reinforces the importance of the orexin system in modulating motivated behaviors and connects HFF to dysfunction of this system. It also identifies the orexin system as a potential contributor to the decreased arousal levels often accompanying obesity (2). Work identifying neural mechanisms linking sleep/ wakefulness and metabolism is urgently required for understanding the growing trend of poor sleep and metabolic disturbances. Shortened sleep durations are becoming increasingly common, are associated with metabolic sequalae, and increase the risk of obesity and diabetes (25). Interestingly, the orexin system has also been implicated as a key neurotransmitter system affected by sleep loss (25). Therefore, the orexin system may be sensitive to disturbances of both sleep and metabolic homeostasis and may serve as a mechanism perpetuating obesity. Together, these data suggest that orexin system dysfunction may underlie multiple physiological and behavioral consequences of HFF and sleep loss and suggest that the orexin system deserves further consideration as a general effector system linking and mediating changes to homeostatic disruptions.
Address correspondence to: Natalie J. Michael, IUCPQ, 2725 chemin Ste-Foy, local Y3150, Québec, Canada, G1V 4G5. Phone: 418.656.8711, ext. 3341; Email: natalie.michael@criucpq.ulaval.ca.

1. Schwartz MW, et al. Central nervous system control of food intake. Nature. 2000;404(6778):661-671.

2. Vgontzas AN, Bixler EO, Tan TL, Kantner D, Martin LF, Kales A. Obesity without sleep apnea is associated with daytime sleepiness. Arch Intern Med.1998;158(12):1333-1337.

3. Sharma S, Fulton S. Diet-induced obesity promotes depressive-like behaviour that is associated with neural adaptations in brain reward circuitry. Int J Obes (Lond). 2013;37(3):382-389.

4. de Lecea L, et al. The hypocretins: hypothalamusspecific peptides with neuroexcitatory activity. Proc Natl Acad Sci U S A. 1998;95(1):322-327.

5. Sakurai T, et al. Orexins and orexin receptors: a family of hypothalamic neuropeptides and G protein-coupled receptors that regulate feeding behavior. Cell. 1998;92(4):573-585.

6. Peyron C, et al. Neurons containing hypocretin (orexin) project to multiple neuronal systems. J Neurosci. 1998;18(23):9996-10015.

7. Chemelli RM, et al. Narcolepsy in orexin knockout mice: molecular genetics of sleep regulation. Cell. 1999;98(4):437-451.

8. Lin L, et al. The sleep disorder canine narcolepsy is caused by a mutation in the hypocretin (orexin) receptor 2 gene. Cell. 1999;98(3):365-376.

9. Nishino S, Ripley B, Overeem S, Lammers GJ, Mignot E. Hypocretin (orexin) deficiency in human narcolepsy. Lancet. 2000;355(9197):39-40.

10. Thannickal TC, et al. Reduced number of hypocretin neurons in human narcolepsy. Neuron. 2000;27(3):469-474.

11. Yamanaka A, et al. Hypothalamic orexin neurons regulate arousal according to energy balance in mice. Neuron. 2003;38(5):701-713.

12. Tsujino N, Sakurai T. Orexin/hypocretin: a neuropeptide at the interface of sleep, energy homeostasis, and reward system. Pharmacol Rev. 2009;61(2):162-176.

13. Cason AM, Smith RJ, Tahsili-Fahadan P, Moorman DE, Sartor GC, Aston-Jones G. Role of orexin/hypocretin in reward-seeking and addic- tion: implications for obesity. Physiol Behav. 2010;100(5):419-428

14. Tan Y, et al. Impaired hypocretin/orexin system alters responses to salient stimuli in obese male mice. JClin Invest. 2020;130(9):4985-4998.

15. Li Y, Gao XB, Sakurai T, van den Pol AN. Hypocretin/Orexin excites hypocretin neurons via a local glutamate neuron-A potential mechanism for orchestrating the hypothalamic arousal system. Neuron. 2002;36(6):1169-1181.

16. Horvath TL, Gao XB. Input organization and plasticity of hypocretin neurons: possible clues to obesity's association with insomnia. Cell Metab. 2005;1(4):279-286.

17. Linehan V, Fang LZ, Parsons MP, Hirasawa M. High-fat diet induces time-dependent synaptic plasticity of the lateral hypothalamus. $\mathrm{Mol}$ Metab. 2020;36:100977.

18. Cristino L, et al. Obesity-driven synaptic remodeling affects endocannabinoid control of orexinergic neurons. Proc Natl Acad Sci U S A. 2013;110(24):E2229-E2238.

19. Elias CF, et al. Chemically defined projections linking the mediobasal hypothalamus and the lateral hypothalamic area. J Comp Neurol. 1998;402(4):442-459.

20. Michael NJ, et al. Melanocortin regulation of histaminergic neurons via perifornical lateral hypothalamic melanocortin 4 receptors. $\mathrm{Mol}$ Metab. 2020;35:100956.

21. Horvath TL, Diano S, van den Pol AN. Synaptic interaction between hypocretin (orexin) and neuropeptide Y cells in the rodent and primate hypothalamus: a novel circuit implicated in metabolic and endocrine regulations. J Neurosci. 1999;19(3):1072-1087.

22. Schöne C, Apergis-Schoute J, Sakurai T, Adamantidis A, Burdakov D. Coreleased orexin and glutamate evoke nonredundant spike outputs and computations in histamine neurons. Cell Rep. 2014;7(3):697-704.

23. Mochizuki T, et al. Orexin receptor 2 expression in the posterior hypothalamus rescues sleepiness in narcoleptic mice. Proc Natl Acad Sci U S A. 2011;108(11):4471-4476.

24. Torrealba F, Riveros ME, Contreras M, Valdes JL. Histamine and motivation. Front Syst Neurosci. 2012;6:51.

25. Knutson KL, Van Cauter E. Associations between sleep loss and increased risk of obesity and diabetes. Ann N Y Acad Sci. 2008;1129:287-304. 